Pacific

Journal of

Mathematics

COMPONENTS OF THE SPACE PARAMETRIZING GRADED GORENSTEIN ARTIN ALGEBRAS WITH A GIVEN HILBERT FUNCTION

Mats Boij 


\title{
COMPONENTS OF THE SPACE PARAMETRIZING GRADED GORENSTEIN ARTIN ALGEBRAS WITH A GIVEN HILBERT FUNCTION
}

\author{
Mats BoiJ
}

\begin{abstract}
We give geometric constructions of families of graded Gorenstein Artin algebras, some of which span a component of the space $\operatorname{Gor}(T)$ parametrizing Gorenstein Artin algebras with a given Hilbert function $T$. This gives a lot of examples where $\operatorname{Gor}(T)$ is reducible. We also show that the Hilbert function of a codimension four Gorenstein Artin algebra can have an arbitrarily long constant part without having the weak Lefschetz property.
\end{abstract}

\section{Introduction.}

In $R=k[x, y]$ and in $R=k[x, y, z]$, the parameter space $\operatorname{Gor}(T)$ of graded Gorenstein Artin quotients of a given Hilbert function $T$ is irreducible for all $T$. The codimension two case is implied by F. S. Macaulay's result that Gorenstein height two ideals in $k[x, y]$ are complete intersections [8], and the fact that the family of graded complete intersection ideals of given generator degrees is irreducible. The codimension three case was proved S. Diesel [4]. A. Iarrobino and V. Kanev [7] have recently shown that in codimension five and higher, Gor $(T)$ can be reducible. In this paper, we will give a large class of examples where $\operatorname{Gor}(T)$ is reducible, in codimension four and higher.

A. Iarrobino and V. Kanev also proved that there is a close relation between graded Gorenstein Artin algebras of codimension three and finite length Cohen-Macaulay sub-schemes of $\mathbf{P}^{2}$. They show that whenever the Hilbert function $T$ is equal to $s$ for at least three degrees, there is a fibration $\operatorname{Gor}(T) \rightarrow \mathfrak{H}(T) \subseteq \operatorname{Hilb}^{s}\left(\mathbf{P}^{2}\right)$ which takes the form $f$ to the initial part of the ideal $\operatorname{ann}_{R}(f)$. We prove that there can be no such results in higher codimensions, by showing that for $r \geq 4$, there are codimension $r$ Gorenstein Artin algebras with arbitrary long constant part in the Hilbert function, whose initial ideal does not define a sub-scheme of length $s$ in $\mathbf{P}^{r-1}$. The way this is done is by exploring the weak Lefschetz property.

Setup 1.1. Let $k$ be an infinite field and let $R=k\left[x_{1}, x_{2}, \ldots, x_{n}\right]$ be the polynomial ring in $n$ variables. Denote by $E$ the injective hull of $k$ over $R$. If $R$ is graded in non-negative degrees with $\operatorname{deg} x_{i}=1$, then $E$ is naturally 
graded in non-positive degrees, $E=E_{0} \oplus E_{-1} \oplus \cdots$. By Matlis duality, the space $\mathbf{P}\left(E_{-c}\right)$ parametrizes graded Gorenstein Artin quotients of $R$ with socle in degree $c$. For a given symmetric Hilbert function $T=\left(t_{0}, t_{1}, \ldots, t_{c}\right)$, we consider the space of forms $f$ in $E$ such that the Gorenstein Artin algebra $R / \operatorname{ann}_{R}(f)$ has Hilbert function $T$ and we denote this space by Gor $(T)$ (cf. A. Iarrobino [6]). Each element $f$ in $E_{-c}$ gives a map $f: R_{c} \longrightarrow k$, by $f(g)=g \circ f \in E_{0} \cong k$. Let $m_{d, c}: R_{d} \times R_{c-d} \rightarrow R_{c}$ denote the multiplication map. Then we have that

$$
\operatorname{Gor}(T)=\left\{f \in E_{-c} \mid \operatorname{rank} f \circ m_{d, c}=t_{d}, \quad \text { for } d=0,1, \ldots, c\right\} .
$$

Thus there is a natural scheme structure on $\operatorname{Gor}(T)$ as a locally closed subscheme of $\mathbf{P}\left(E_{-c}\right)$ given by the minors of catalecticant matrices. The tangent space to the affine cone over $\operatorname{Gor}(T)$ at a point $f$ in $E_{-c}$ is given by $\operatorname{ann}_{E}\left(\operatorname{ann}_{R}(f)^{2}\right)_{-c}($ cf. A. Iarrobino and V. Kanev [7]).

\section{Points on rational normal curves.}

In this section we will construct families of Gorenstein Artin algebras related to sets of points on rational normal curves. We will compute the dimension of these families, as well as the dimension of the tangent space to $\operatorname{Gor}(T)$ at a special point in each family. In codimension four, this point is non-singular, and the dimension of the tangent space coincides with the dimension of the family, which shows that the family in this case spans an irreducible component of $\operatorname{Gor}(T)$.

Setup 2.1. For a positive integer $n$, let

$$
\mathfrak{T}_{n}=\left\{C \mid C \text { is rational normal curve in } \mathbf{P}^{n}\right\} .
$$

This is an irreducible open subset of the Hilbert scheme $\operatorname{Hilb}^{n x+1}\left(\mathbf{P}^{n}\right)$ parametrizing curves in $\mathbf{P}^{n}$ of Hilbert polynomial $n x+1$. The dimension of $\mathfrak{T}_{n}$ is $(n+1)^{2}-4=(n+3)(n-1)$. Denote by $R$ the homogeneous coordinate ring of $\mathbf{P}^{n}$.

Definition 2.2. For integers $n$ and $s$, let

$$
\overline{\mathfrak{P}}_{n, s}=\left\{(X, C) \mid I_{C} \subseteq I_{X}\right\} \subseteq \operatorname{Hilb}^{s}\left(\mathbf{P}^{n}\right) \times \mathfrak{T}_{n} .
$$

Let $\mathfrak{P}_{n, s} \subseteq \overline{\mathfrak{P}}_{n, s}$ be the open subset on which the Hilbert function is maximal.

Lemma 2.3. For non-negative integers $n$ and $s$, we have that:

i) The set $\overline{\mathfrak{P}}_{n, s}$ is closed and irreducible of dimension $(n+3)(n-1)+s$.

ii) If $s \geq 2 n+1$, the induced map $\mathfrak{P}_{n, s} \longrightarrow \operatorname{Hilb}^{s}\left(\mathbf{P}^{n}\right)$ is injective.

Proof. Since $\mathfrak{T}_{n}$ is irreducible, $\operatorname{Hilb}^{s}\left(\mathbf{P}^{n}\right)$ is proper and all the fibers over $\mathfrak{T}_{n}$ are irreducible of the same dimension $s$, we have that $\overline{\mathfrak{P}}_{n, s}$ is irreducible of dimension $(n+3)(n-1)+s$, by Lemma 5.1 . 
A general element of $\mathfrak{P}_{n, s}$ is a set $X$ of $s$ distinct points on a specified rational normal curve $C$ in $\mathbf{P}^{n}$. For an element $(X, C)$ in $\mathfrak{P}_{n, s}$ with $s \geq$ $2 n+1$, the set $X$ determines the curve $C$, since the ideal of the curve is generated in degree 2 and there are no other generators of $I_{X}$ in degree 2 . Thus, for $s \geq 2 n+1$, we get an injective map from $\mathfrak{P}_{n, s}$ to the Hilbert scheme $\operatorname{Hilb}^{s}\left(\mathbf{P}^{n}\right)$ parametrizing length $s$ sub-schemes of $\mathbf{P}^{n}$.

Definition 2.4. For integers $n, s$ and $c$, let

$$
\overline{\mathfrak{P F}}_{n, s, c}=\left\{(f, X, C) \mid f \in \operatorname{ann}_{E}\left(I_{X}\right)\right\} \subseteq \mathbf{P}\left(E_{-c}\right) \times \mathfrak{P}_{n, s} .
$$

Let $\mathfrak{P F}_{n, s, c}$ be the open subset of $\overline{\mathfrak{P F}}_{n, s, c}$ on which the Hilbert function of the Gorenstein quotient $R / \operatorname{ann}_{R}(f)$ is maximal.

Lemma 2.5. For non-negative integers $n, s$ and $c$, we have that:

i) The set $\overline{\mathfrak{P F}}_{n, s, c}$ is closed and irreducible of dimension

$$
(n+3)(n-1)+s+H_{X}(c)-1 .
$$

ii) If $H_{X}(\lfloor(c-1) / 2\rfloor)=s$ and $s \geq 2 n+1$, then the induced map

$$
\mathfrak{P F}_{n, s, c} \longrightarrow \mathbf{P}\left(E_{-c}\right) \quad \text { is injective. }
$$

iii) If $(f, X, C)$ is in $\mathfrak{P F}_{n, s, c}$, then the Hilbert function of the Gorenstein Artin algebra $A=R / \operatorname{ann}_{R}(f)$ is given by

$$
H_{A}(d)=\min \{n d+1, s, n(c-d)+1\}, \quad \text { for } d=0,1, \ldots, c .
$$

Proof. Here we have that $\mathfrak{P}_{n, s}$ is irreducible, $\mathbf{P}\left(E_{-c}\right)$ is proper, and all the fibers are projective spaces of the same dimension $H_{X}(c)-1$. Hence we get that $\mathfrak{P F}_{n, s, c}$ is irreducible of dimension $(n+3)(n-1)+s+H_{X}(c)-1$, by Lemma 5.1.

Since a general element $(f, X, C)$ of $\mathfrak{P F}_{n, s, c}$ corresponds to a reduced set of points $X$ on a rational normal curve $C$ together with a general form $f$ annihilating the ideal of the points, we have that the Hilbert function of the quotient $A=R / \operatorname{ann}_{R}(f)$ is given by

$$
H_{A}(d)=\min \left\{H_{X}(d), H_{X}(c-d)\right\}, \quad \text { for } d=0,1, \ldots, c .
$$

If $H_{X}(\lfloor(c-1) / 2\rfloor)=s$, we have that the generators of $I_{X}$ can be in no higher degree than $\lfloor(c-1) / 2\rfloor+1=\lfloor(c+1) / 2\rfloor$. In that case we get that $H_{A}(\lfloor(c+$ $1) / 2\rfloor)=H_{A}(\lfloor(c-1) / 2\rfloor)=s$ and $\operatorname{ann}_{R}(f) / I_{X}$ has no generators in degrees lower than $\lfloor(c+1) / 2\rfloor+1$. Hence the Gorenstein ideal $\operatorname{ann}_{R}(f)$ determines the ideal $I_{X}$. If in addition $s \geq 2 n+1$, we get, by the previous lemma, an injective map from an open set in $\mathfrak{P F}_{n, s, c}$ into $\mathbf{P}\left(E_{-c}\right)$ by projection onto the first factor.

Definition 2.6. Denote by $\mathfrak{F}_{n, s, c}$ the image of $\mathfrak{P F}_{n, s, c}$ in $\mathbf{P}\left(E_{-c}\right)$ when $s \geq$ $2 n+1$ and $H_{X}(\lfloor(c-1) / 2\rfloor)=s$. 
Proposition 2.7. For positive integers $n, s$ and $c$ with $2 n+1 \leq s \leq n\lfloor(c-$ 1)/2」 +1 , we have that:

i) The family $\mathfrak{F}_{n, s, c}$ is irreducible of dimension $(n+3)(n-1)+2 s-1$.

ii) Let $f$ be an element of $\mathfrak{F}_{n, a, c}$, then the Hilbert function of $A=$ $R / \operatorname{ann}_{R}(f)$ is given by

$$
H_{A}(d)=\min \{n d+1, s, n(c-d)+1\}, \quad \text { for } d=0,1, \ldots, c .
$$

Proof. The proposition is an immediate consequence of Lemma 2.5 since the condition $H_{X}(\lfloor(c-1) / 2\rfloor)=s$ is equivalent to $n\lfloor(c-1) / 2\rfloor+1 \geq s$.

Definition 2.8. Denote by $T_{n, s, c}$ the Hilbert function given in Proposition 2.7.

Remark 2.9. If $f$ is a general element of $\mathfrak{F}_{n, s, c}$, and $\lfloor c / 2\rfloor n+1 \geq s$, then the Gorenstein Artin algebra $R / \operatorname{ann}_{R}(f)$ satisfies the weak Lefschetz property (cf. §3.1). Indeed, the ideal is $\operatorname{ann}_{R}(f)=I_{X}+J$, where $J$ is generated in degree at least $\lfloor c / 2\rfloor$, and since $I_{X}$ is the defining ideal of a finite set of points, $R / I_{X}$ has a non-zero divisor.

2.1. The tangent space to Gor $(T)$. We will now compute the tangent space of $\operatorname{Gor}(T)$ at special point of the family $\mathfrak{F}_{n, s, c}$. In fact, the point we will pick will not even be in the family, strictly speaking, but in the closure. However, it will have the same Hilbert function. Thus the dimension of the tangent space at this point will give an upper bound for the dimension of the tangent space at a general point of the family $\mathfrak{F}_{n, s, c}$.

We now identify $E$ with the polynomial ring $k\left[X_{0}, X_{1}, \ldots, X_{n}\right]$, where $R$ acts on $E$ by contraction, i.e., $x_{i} \circ X_{j}=\delta_{i j}$.

Lemma 2.10. For integers $2 \leq a_{1} \leq a_{2}, \cdots \leq a_{n}$, such that $a_{n}-a_{1} \leq 1$, and $c \geq 2 a_{n}+2$, let $F=\sum_{i=1}^{n} X_{0}^{a_{i}} X_{i}^{c-a_{i}}$ and let $A=R / \operatorname{ann}_{R}(F)$. Then the following hold:

i) The dimension of $\operatorname{ann}_{E}\left(\operatorname{ann}_{R}(F)^{2}\right)_{-c}$ is $2 n^{2}+2 \sum_{i=1}^{n} a_{i}$.

ii) The Hilbert function of $A$ is

$$
H_{A}(d)=\min \left\{n d+1, n+\sum_{i=1}^{n} a_{i}, n(c-d)+1\right\}, \quad \text { for } d=0,1, \ldots, c .
$$

Proof. The ideal $I=\operatorname{ann}_{R}(F)$ is generated by the elements

$$
\begin{cases}x_{i} x_{j}, & \text { for } 1 \leq i<j \leq n \\ x_{0}^{a_{n}+1} & \text { for } 1 \leq i \leq n \\ x_{i} x_{0}^{a_{i}+1}, & \\ x_{n}^{c+1-a_{n}} & \text { for } 1 \leq i \leq n . \\ x_{i}^{c-a_{i}}-x_{n}^{c-a_{n}} x_{0}^{a_{n}-a_{i}},\end{cases}
$$


Denote by $I_{C}$ the ideal $\left(\left\{x_{i} x_{j}\right\}_{1 \leq i<j \leq n}\right)$, and by $I_{X}$ the ideal $I_{C}+\left(x_{0}^{a_{n}+1}\right.$, $\left.\left\{x_{i} x_{0}^{a_{i}+1}\right\}_{i=1}^{n}\right)$. We have that the only monomials in $X_{0}, X_{1}, \ldots, X_{n}$ of degree $c$ that annihilate $I_{X}^{2}$ are

$$
\begin{cases}X_{i}^{c}, X_{i}^{c-1} X_{0}, \ldots, X_{i}^{c-2 a_{i}-1} X_{0}^{2 a_{i}+1}, & \text { for } 1 \leq i \leq n \\ X_{j} X_{i}^{c-1}, X_{j} X_{i}^{c-2} X_{0}, \ldots, X_{j} X_{i}^{c-1-a_{i}} X_{0}^{a_{i}} & \text { for } 1 \leq i \neq j \leq n .\end{cases}
$$

Since $c \geq 2 a_{n}+2$, these are all distinct. The number of monomials of the first kind is $2 \sum_{i=1}^{n}\left(a_{i}+1\right)$, and all of them actually annihilate $\operatorname{ann}_{R}(F)^{2}$.

Now, for a linear combination of the second kind of monomials to annihilate the products $x_{i} x_{j}\left(x_{i}^{c-a_{i}}-x_{n}^{c-a_{n}} x_{0}^{a_{n}-a_{i}}\right)$, the only terms involving $X_{i} X_{j}$ that can occur are

$$
X_{j} X_{i}^{c-a_{i}} X_{0}^{a_{i}-1}, X_{j} X_{i}^{c-a_{i}-1} X_{0}^{a_{i}}, \quad 1 \leq i, j \leq n, \quad i \neq j .
$$

The number of such terms is $2 n(n-1)$. Note that since $a_{i} \geq 2$ for all $i$, the elements $x_{i} x_{i}\left(x_{i}^{c-a_{i}}-x_{n}^{c-a_{n}} x_{0}^{a_{n}-a_{i}}\right)$ all lie in degree at most $c$.

Since $a_{n}-a_{1} \leq 1$, the product of a generator $x_{i} x_{0}^{a_{i}+1}$, or $x_{0}^{a_{n}+1}$ with a generator $x_{i}^{c-a_{i}}-x_{n}^{c-a_{n}} x_{0}^{a_{n}-a_{i}}$ or $x_{n}^{c+1-a_{n}}$ always has higher degree than $c$. Thus the annihilator of $\operatorname{ann}_{R}(F)^{2}$ is generated by the $2 \sum_{i=1}^{n}\left(a_{i}+1\right)+$ $2 n(n-1)=2 n^{2}+2 \sum_{i=1}^{n} a_{i}$ distinct monomials given above.

The Hilbert function of $R / I_{C}$ is $H_{C}(d)=n d+1$, for $d \geq 0$ and the Hilbert function of $R / I_{X}$ is $H_{X}(d)=\min \left\{n d+1, n+\sum_{i=1}^{n} a_{i}\right\}$, for $d \geq 0$. The latter is a consequence of the fact that $I_{X} / I_{C}$ is generated in degrees at least $a_{n}+1$, and for $d>a_{n}$, we have that $\left\{x_{i}^{d}, x_{i}^{d-1} x_{0}, \ldots, x_{i}^{d-a_{i}} x_{0}^{a_{i}}\right\}_{1 \leq i \leq n}$ is a $k$-basis of $\left(R / I_{X}\right)_{d}$. Since there are no generators of $\operatorname{ann}_{R}(F) / I_{X}$ in degrees lower than $c-a_{n} \geq c / 2+1$, we have that the Hilbert function of $A=R / \operatorname{ann}_{R}(F)$ is $H_{A}(d)=\min \left\{n d+1, n+\sum_{i=1}^{n} a_{i}, n(c-d)+1\right\}$, for $d=0,1, \ldots, c$.

Proposition 2.11. Given positive integers $n, s, c$, write $s=p n+q$, where $1 \leq q \leq n$. Let

$$
F=\sum_{i=1}^{n-q} X_{0}^{p-1} X_{i}^{c+1-p}+\sum_{i=n-q+1}^{n} X_{0}^{p} X_{i}^{c-p} .
$$

If $c \geq 2\lceil s / n\rceil$ and $s \geq 3 n$, then we have that:

i) $R / \operatorname{ann}_{R}(F)$ has Hilbert function $T_{n, s, c}$.

ii) The tangent space to $\operatorname{Gor}\left(T_{n, s, c}\right)$ at $F$ has dimension $2 n(n-1)+2 s-1$.

iii) F lies in the closure of $\mathfrak{F}_{n, s, c}$.

Proof. We apply Lemma 2.10 with

$$
a_{i}= \begin{cases}p-1, & \text { for } 1 \leq i \leq n-q \\ p, & \text { for } n-q<i \leq n\end{cases}
$$


We have that $s \geq 3 n$ implies that $a_{1} \geq 2$. Furthermore, $a_{n}=p=\lfloor(s-$ 1) $/ n\rfloor=\lceil s / n\rceil-1$, which shows that $c \geq 2\lceil s / n\rceil$ implies that $c \geq 2 a_{n}+2$. Thus the assumptions of the lemma are fulfilled and the first two assertions follow directly from the lemma.

To prove the third assertion, we look at the ideals $I_{C}$ and $I_{X}$ from the proof of Lemma 2.10. The geometric picture is that $C$ is the union of $n$ lines through one point and $X$ is a scheme of length $s$ concentrated at one point of each line, with multiplicity $p-1$ at $n-q$ points and multiplicity $p$ at the remaining $q$ points. This curve $C$ does not belong to the family $\mathfrak{T}_{n}$ of rational normal curves, but it has the right Hilbert function, and it is in the closure of $\mathfrak{T}_{n}$ in the Hilbert scheme $\operatorname{Hilb}^{n x+1}\left(\mathbf{P}^{n}\right)$. In fact, we can deform the ideal of $C$ into a family of rational normal curves by looking at the ideal of $2 \times 2$-minors of the matrix

$$
\left(\begin{array}{cccc}
\alpha x_{0}+x_{1} & \alpha x_{1}+2 x_{2} & \ldots & \alpha x_{n-1}+n x_{n} \\
x_{1} & x_{2} & \ldots & x_{n}
\end{array}\right)
$$

where $\alpha$ is a parameter. When $\alpha=0$, we get the ideal $I_{C}$, while for all but possibly finitely many values of $\alpha$, we get the ideal of a rational normal curve. When we construct the family $\mathfrak{P}_{n, s}$ over the component of the Hilbert scheme $\operatorname{Hilb}^{n x+1}\left(\mathbf{P}^{n}\right)$ that contains $\mathfrak{T}_{n}$, we will no longer get irreducible fibers over all points. However, we can take the closure of the family $\mathfrak{P}_{n, s}$ in $\operatorname{Hilb}^{s}\left(\mathbf{P}^{n}\right) \times \operatorname{Hilb}^{n x+1}\left(\mathbf{P}^{n}\right)$ to get an irreducible family. Then we will get a family that contains $(X, C)$, since the support of $X$ is away from the singularity of $C$. We can then continue the construction to get a slightly larger family that contains $\mathfrak{F}_{n, s, c}$ as an open dense subset. This family also contains $F$, which concludes the proof.

Corollary 2.12. For $n=3, s \geq 9$, and $\lfloor(c-1) / 2\rfloor \geq\lceil s / 3\rceil$ the family $\mathfrak{F}_{3, s, c}$ spans a component of $\operatorname{Gor}\left(T_{3, s, c}\right)$.

Proof. In this case, we have that the dimension of the tangent space is $11+2 s$, which coincides with the dimension of the family $\mathfrak{F}_{3, s, c}$. Thus the point we have chosen is, in fact, a smooth point of $\operatorname{Gor}\left(T_{3, s, c}\right)$, and the family $\mathfrak{F}_{3, s, c}$ must span an irreducible component. It remains to check that the assumptions of Propostion 2.7 and Proposition 2.11 apply. The condition $s \geq 9$ is equivalent to $s \geq 3 n$, and the condition $\lfloor(c-1) / 2\rfloor \geq\lceil s / 3\rceil$ implies that $c \geq 2\lceil s / n\rceil$ and that $n\lfloor(c-1) / 2\rfloor+1 \geq s$.

\section{Families of Gorenstein Artin algebras not satisfying the weak Lefschetz property.}

In this section we give a construction of families $\mathfrak{G}_{n, c}$ of Gorenstein Artin algebras not satisfying the weak Lefschetz property. The algebras in the families $\mathfrak{G}_{n, c}$ are generalizations of the examples given by R. Stanley [10] 
and by D. Bernstein and A. Iarrobino [2] showing the existence of nonunimodal Hilbert functions of Gorenstein algebras. In addition, the Hilbert functions of the algebras in $\mathfrak{G}_{n, c}$ are given by $T_{n, c+2, c}$. We will use this fact to prove that $\operatorname{Gor}\left(T_{n, c+2, c}\right)$ is reducible for most choices of $n$ and $c$. The family $\mathfrak{G}_{n, c}$ consists of Gorenstein ideals whose initial part is the square of the ideal of a line.

Setup 3.1. For a positive integer $n$, let $\mathbf{G}(1, n)$ be the grassmannian of lines in $\mathbf{P}^{n}$. It is an irreducible variety of dimension $2(n-1)$. For integers $n$ and $c$, let

$$
\overline{\mathfrak{L}}_{n, c}=\left\{(f, L) \mid f \in \operatorname{ann}_{E}\left(I_{L}^{2}\right)\right\} \subseteq \mathbf{P}\left(E_{c}\right) \times \mathbf{G}(1, n) .
$$

Let $\mathfrak{L G}_{n, c}$ be the open subset of $\overline{\mathfrak{L} G}_{n, c}$ where the Hilbert function is maximal.

Proposition 3.2. For positive integers $n$ and $c$ we have that:

i) The set $\mathfrak{L G}_{n, c}$ is irreducible of dimension $2(n-1)+n c$.

ii) For $(f, L)$ in $\mathfrak{L G}_{n, c}$, the Hilbert function of $A=R / \operatorname{ann}_{R}(f)$ is given by

$$
H_{A}(d)=\min \{n d+1, c+2, n(c-d)+1\},
$$

for $d=0,1, \ldots, c$.

iii) If $c \geq 2 n-1$, the map $\mathfrak{L G}_{n, c} \longrightarrow \mathbf{P}\left(E_{-c}\right)$ is injective.

Proof. The map $\overline{\mathfrak{L G}}_{n, c} \longrightarrow \mathbf{G}(1, n)$ is proper and surjective, $\mathbf{G}(1, n)$ is irreducible of dimension $2(n-1)$ and all the fibers are irreducible of dimension $n c$. The latter since the Hilbert function of $R / I_{L}^{2}$ is $n d+1$. Hence the set $\overline{\mathfrak{L}}_{n, c}$ is irreducible of dimension $2(n-1)+n c$, by Lemma 5.1.

To prove the second assertion, we note that we can change variables so that the line $L$ is given by the ideal $\left(x_{2}, x_{3}, \ldots, x_{n}\right)$. Let $H_{A}(d)$ denote the Hilbert function of $A=R / \operatorname{ann}_{R}(f)$. Then $H_{A}(d)$ is given by the dimension of $R_{d} \circ f$. The condition that $f$ annihilates $I_{L}^{2}$, shows that

$$
R_{d} \circ f=\left[\left(x_{0}, x_{1}\right)^{d}\right]_{d} \circ f+\left[\left(x_{0}, x_{1}\right)^{d-1}\left(x_{2}, x_{3}, \ldots, x_{n}\right)\right]_{d} \circ f .
$$

Thus we get $H_{A}(d) \leq d+1+d(n-1)$. Since the latter term is contained in $\operatorname{ann}_{E}\left(x_{2}, \ldots, x_{n}\right) \cap E_{d-c}$, we also get $H_{A}(d) \leq d+1+c-d+1=c+2$. Since the $H_{A}(d)$ is symmetric around $d=c / 2$, we get that the Hilbert function in the proposition is an upper bound for the $H(d)$.

It now suffices to show that there is one $f$ that gives this maximal Hilbert function. This is can be done by choosing a compressed Artin level algebra $B$ in two variables with socle of dimension $n-1$ in degree $c-1$, i.e., $B$ is a level algebra with Hilbert function $H_{B}(d)=\min \{d+1,(c-d)(n-1)\}$, and take $A=B \times B^{\curlyvee}$ - the trivial extension of $A$ by its dual.This Gorenstein algebra has the right Hilbert function, (cf. D. Bernstein and A. Iarrobino [2], or M. Boij and D. Laksov [3]), and the square of the ideal generated by the generators of $B^{\sim}$ is zero in $A$. 
For the third assertion, we need to prove that if $c \geq 2 n-1$ and $(f, L)$ is in $\mathfrak{G} \mathfrak{L}_{n, c}$ then the ideal $\operatorname{ann}_{R}(f)$ determines the line $L$. The ideal $I_{L}^{2}$ is generated in degree 2, and looking at the Hilbert function, we see that $\operatorname{ann}_{R}(f)$ has no other generators in degree 2. Thus the line is determined by $\operatorname{ann}_{R}(f)$.

Definition 3.3. Denote the image of $\mathfrak{L G}_{n, c}$ in $\mathbf{P}\left(E_{-c}\right)$ by $\mathfrak{G}_{n, c}$, for $c \geq$ $2 n-1$.

3.1. The weak Lefschetz property. Recall that a Gorenstein Artin algebra $A$ with socle in degree $c$ satisfies the weak Lefschetz property if the multiplication maps $\ell: A_{d} \rightarrow A_{d+1}$, for $d=0,1,\lfloor(c-1) / 2\rfloor$, are injective for a general linear form $\ell \in A_{1}$. We will use the following characterization of the weak Lefschetz property, due to J. Watanabe [11, Prop. 3.4].

Proposition 3.4. The Gorenstein Artin algebra $A=R / \operatorname{ann}_{R}(f)$ satisfies the weak Lefschetz property if and only if the Hilbert function of $R / \operatorname{ann}_{R}(\ell \circ$ $f)$ agrees with the Hilbert function of $A$ in degrees less than $c / 2$ for a general linear form $\ell \in R$.

Proposition 3.5. Suppose that $n \geq 3$, and $c=5$ or $c \geq 7$, then for an element $f \in \mathfrak{G}_{n, c}$ the Gorenstein Artin quotient $A=R / \operatorname{ann}_{R}(f)$ does not satisfy the weak Lefschetz property.

Proof. Under the assumptions of the proposition, the Hilbert function of the Gorenstein Artin algebra $A=R / \operatorname{ann}_{R}(f)$ for an element $f$ of $\mathfrak{G}_{n, c}$ attains the value $c+2$ in degree $\lfloor(c-1) / 2\rfloor$. On the other hand, for any linear form $\ell \in R$, we have that $\ell \circ f$ is in $\mathfrak{G}_{n, c-1}$, but by Proposition 3.2, we have that the Hilbert function of $R / \operatorname{ann}_{R}(\ell \circ f)$ is at most $c+1$. Thus it cannot agree with the Hilbert function of $R / \operatorname{ann}_{R}(f)$ in degree $\lfloor(c-1) / 2\rfloor$, and the proposition follows from Proposition 3.4.

A. Iarrobino and V. Kanev [7] have shown that for codimension three Gorenstein algebras there is a strong connection to subschemes of $\mathbf{P}^{2}$. In particular, they show that if a codimension three Gorenstein Artin algebra has Hilbert function containing a subsequence $(s, s, s)$, there is a uniquely determined Cohen-Macaulay subscheme of $\mathbf{P}^{2}$ - the annihilating schemewhich ideal is generated by the lower degree part of the Gorenstein ideal. In particular, this means that the Gorenstein algebra must have the weak Lefschetz property. The following theorem shows that a similar result is impossible in codimension four and higher.

Theorem 3.6. In codimension four and higher, there are graded Gorenstein Artin algebras not satisfying the weak Lefschetz property with arbitrarily long constant part in the Hilbert function. 
Proof. By Proposition 3.5 the algebras in $\mathfrak{G}_{n, c}$ do not have the weak Lefschetz property. However, their Hilbert function have a constant subsequence $(c+2, c+2, \ldots, c+2)$, of length $c+1-2\lfloor(c+1) / n\rfloor \geq(c+1)(1-2 / n)$. For $n \geq 3$ this can be arbitrarily large.

\section{Components of $\operatorname{Gor}(T)$.}

We can now use the results from the previous sections to conclude that in many cases, we get two distinct components of $\operatorname{Gor}(T)$. The idea is that the families $\mathfrak{F}_{n, c+2, c}$ and $\mathfrak{G}_{n, c}$ both parametrize Gorenstein Artin algebras with Hilbert function $T_{n, c+2, c}$ while the latter family, for large $c$, has higher dimension than the dimension of the tangent space of the first family, so it cannot lie in the same component.

Theorem 4.1. For $n \geq 3$ and $c \geq \max \{11,3 n-2\}$ the parameter space $\operatorname{Gor}\left(T_{n, c+2, c}\right)$ is reducible with one component containing $\mathfrak{F}_{n, c+2, c}$ and another component containing $\mathfrak{G}_{n, c}$.

Proof. For $c \geq 2\lceil(c+2) / n\rceil$ and $c+2 \geq 3 n$ the tangent space of $\operatorname{Gor}\left(T_{n, c+2, c}\right)$ at a general point of $\mathfrak{F}_{n, c+2, c}$ has dimension at most $2 n(n-1)+2(c+$ $2)-1$ according to Proposition 2.11. Thus the irreducible component of $\operatorname{Gor}\left(T_{n, c+2, c}\right)$ that contains $\mathfrak{F}_{n, c+2, c}$ has at most this dimension.

For $c \geq 2 n-1$ the dimension of $\mathfrak{G}_{n, c}$ is $2(n-1)+n c$, by Proposition 3.2. Any component of $\operatorname{Gor}\left(T_{n, c+2, c}\right)$ that contains $\mathfrak{G}_{n, c}$ and some element with the weak Lefschetz property must have dimension at least $2 n-1+n c$, since this is an open property.

One can easily check that $c \geq \max \{11,3 n-2\}$ implies that $c \geq 2\lceil c+2 / n\rceil$, $c+2 \geq 3 n$ and $c \geq 2 n-1$. Furthermore, $2 n-1+n c>2 n(n-1)+2(c+2)-1$, which implies that the family $\mathfrak{G}_{n, c}$ cannot be in the same component of $\operatorname{Gor}\left(T_{n, c+2, c}\right)$ as $\mathfrak{F}_{n, c+2, c}$.

Remark 4.2. The smallest example of reducible Gor $(T)$ that is given by Theorem 4.1 is $n=3, c=11$, which gives Hilbert function

$$
T=(1,4,7,10,13,13,13,13,10,7,4,1) .
$$

In this case we have that both families $\mathfrak{F}_{3,13,11}$ and $\mathfrak{G}_{3,11}$ have dimension 37 , and by computer calculations that the tangent space of $\operatorname{Gor}(T)$ at a general point of $\mathfrak{G}_{3,11}$ has dimension 37 , so the family spans its component in this case.

By means of the computer algebra system, Macaulay [1], we have checked that the dimension of the tangent space of $\operatorname{Gor}\left(T_{3, c+2, c}\right)$ at a general point of $\mathfrak{G}_{3, c}$ coincides with the dimension of $\mathfrak{G}_{3, c}$ for $c=11,12, \ldots, 34$. 


\section{Appendix.}

In proving the irreducibility of the families $\mathfrak{F}_{n, s, c}$ and $\mathfrak{G}_{n, c}$ we needed the following lemma. This is stated in the projective case in Shafarevich $[\mathbf{9}$, Thm. 8, Chap. I], although the proof there uses only properness. For convenience, we give a brief proof.

Lemma 5.1. Let $f: X \longrightarrow Y$ be a proper surjective morphism of schemes of finite type over $k$. Suppose that $Y$ is irreducible of dimension $d_{Y}$ and that all the fibers of $f$ are irreducible of dimension $d$. Then $X$ is irreducible of dimension $d+d_{Y}$.

Proof. Firstly, we may assume that both $X$ and $Y$ are reduced. Thus we can use R. Hartshorne [5, Ch. II, Ex. 3.22] on the restrictions of $f$ to the components of $X$ that surject onto $Y$. Since $f$ is proper and surjective and $Y$ is irreducible each component of $X$ must either map onto $Y$ or outside an open dense subset $U$ of $Y$.

For each component $X^{\prime}$ of $X$ surjecting onto $Y$ there is an open set in $Y$ over which the fibers of $\left.f\right|_{X^{\prime}}$ have minimal dimension. Let $y$ be a point in the intersection of all such open sets and $U$. Since the fiber $X_{y}$ is irreducible, it must lie completely within at least one of the fibers $X_{y}^{\prime}$. Hence, the fibers of the map $X^{\prime} \longrightarrow Y$ must all have dimension $d$, and since the fibers are irreducible, we must have that $X_{y^{\prime}}^{\prime}=X_{y^{\prime}}$ for all points $y^{\prime}$ in $Y$. Thus $X=X^{\prime}$ is irreducible of dimension $d+d_{Y}$.

\section{References}

[1] D. Bayer and M. Stillman, Macaulay: A system for computation in algebraic geometry and commutative algebra, Source and object code available for Unix and Macintosh computers. Contact the authors, or download from zariski . harvard.edu via anonymous ftp.

[2] D. Bernstein and A. Iarrobino, A nonunimodal graded Gorenstein Artin algebra in codimension five, Comm. Algebra, 20(8) (1992), 2323-2336.

[3] M. Boij and D. Laksov, Nonunimodality of graded Gorenstein Artin algebras, Proc. Amer. Math. Soc., 120(4) (1994), 1083-1092.

[4] S.J. Diesel, Irreducibility and dimension theorems for families of height 3 Gorenstein algebras, Pacific J. Math., 172(2) (1996), 365-397.

[5] R. Hartshorne, Algebraic geometry, Vol. 52 of Graduate Texts in Mathematics, Springer-Verlag, New York-Heidelberg-Berlin, 1977.

[6] A. Iarrobino, Associated graded algebra of a Gorenstein Artin algebra, Vol. 514 of Memoirs of the American Mathematical Society, American Mathematical Society, Providence, 1994.

[7] A. Iarrobino and V. Kanev, The length of a homogeneous form, determinantal loci of catalecticants, and Gorenstein algebras, preprint, 1996. 
[8] F.S. Macaulay, The algebraic theory of modular systems, Cambridge Mathematical Library, Cambridge University Press, Cambridge, 1994. Revised reprint of the 1916 original.

[9] I.R. Shafarevich, Basic algebraic geometry 1, Springer-Verlag, Berlin, second edition, 1994. Varieties in projective space.

[10] R. Stanley, Hilbert functions of graded algebras, Adv. in Math., 28 (1978), 57-83.

[11] J. Watanabe, The Dilworth number of Artinian rings and finite posets with rank function, in 'Commutative algebra and combinatorics' (Kyoto, 1985), Vol. 11 of Adv. Stud. Pure Math., 303-312, North-Holland, Amsterdam, 1987.

Received July 9, 1997 and revised September 12, 1997.

\section{$\mathrm{KTH}$}

S-100 44 Stockholm

SWEDEN

E-mail address: boij@math.kth.se 